\title{
Effects of Hip Thrust Training on the Strength and Power Performance in Collegiate Baseball Players
}

\author{
Kun-Han Lin ${ }^{1}$, Chih-Min Wu ${ }^{2}$, Yi-Ming Huang ${ }^{3}$ and Zong-Yan Cai ${ }^{4}$ \\ 1. Physical Education Office, National Tsing Hua University, Hsin Chu City 300, Taiwan \\ 2. Department of Leisure and Sports Management, Cheng Shiu University, Kaohsiung City 833, Taiwan \\ 3. Optimum Kinetics Institute, Kaohsiung City 801, Taiwan \\ 4. Division of Physical and Health Education, Center for General Education, National Sun Yat-sen University, Kaohsiung City 804, \\ Taiwan
}

\begin{abstract}
The aim of the present study was to investigate the effects of hip thrust (HT) training on the strength and power performance of lower extremities. In total, 20 male collegiate baseball players participated in this study and were divided into a HT training group (HTT) and a control group (CON). Participants in the HTT group added HTT movements to a regular baseball training regimen (3 times per week for 8 weeks), whereas participants in the CON group only performed the regular baseball training regimen. Before and after the training sessions, strength performance was assessed via tests of squat strength and 3 repetition maximum (3RM) HT strength; power performance was assessed via vertical jump height, standing long jump distance, and 30-m sprint time. The results indicated significant improvement in squat strength $(31 \% \pm 15 \%)$ and 3RM HT strength $(36 \% \pm 16 \%)(P<0.05)$, but not in vertical jump height, standing long jump distance, and 30-m sprint time. Furthermore, the improvement in squat strength highly correlated with 3RM HT strength $(\mathrm{r}=0.83, P<0.05)$. In conclusion, HTT plays a role in developing squat strength but does not appear to improve power performance.
\end{abstract}

Key words: Hip extension, squat, vertical jump, standing long jump, sprint.

\section{Introduction}

The hip joint is the biggest and most powerful joint in the lower extremity. The flexion-extension movement of the hip plays a crucial role in most daily activities, such as squatting, standing up, walking, climbing, jumping, and running $[1,2]$ and participates in the control of trunk movements [3]. Having a strong hip joint is important because weak hip strength is related to back pain $[4,5]$ and knee injury $[6,7]$. Poor hip strength also correlates with proneness to fatigue in patients with chronic lower-back pain in comparison to healthy control subjects during a sustained back extension endurance test [3]. In addition to meeting the demands of daily activity, hip extension movement is becoming more attractive in sports training as it is

Corresponding author: Zong-Yan, Cai, Ph.D., assiatance professor, research fields: exercise physiology, exercise biochemistry. important for sprinting, jumping, and various athletic movement patterns [1, 8-11].

Hip thrust (HT) exercise, introduced by Contreras and colleagues, is an emerging strength training method to enhance hip extension strength [8]. The HT training (HTT) technique is characterized by increasing the resistance with an elastic band or a barbell across the hips during a bridging exercise [8]. The regular training of the loaded hip extension moment created by HT is theorized to enhance horizontal force production and maximize gluteus hypertrophy [8-10]. It was hypothesized that the increased hip strength performance subsequently transfers to sprint, vertical jump, standing long jump, and squat capacity, which play crucial roles in the performance of athletes [11].

Performing hip extension exercise regularly (e.g., kettlebell swing) has previously been reported to facilitate squat strength and explosive performance 
[12]. However, studies using the HTT method to improve these two areas of performance are limited. Contreras et al. [13] compared the effects of 6-week HTT versus a front-squat resistance training program on performance in adolescent males. In their study, a within-group effect on HT 3 repetition maximum (3RM) strength, front squat strength, vertical jump, horizontal jump, and 10-20 $\mathrm{m}$ sprint times showed improvement. However, due to the purpose of their study, comparing the effects of two intervention training methods (HT vs. front squat), there was no control group in this study. Additionally, their subjects were adolescents; the training effect may have limited application to other populations.

Therefore, this study was undertaken to specifically investigate the effects of HTT on the strength and power performance of lower extremities in experienced athletes.

\section{Methods}

\subsection{Study design}

The study was designed as a randomized controlled trial. Recreational college baseball players were randomly assigned to a HTT group and a control $(\mathrm{CON})$ group. Subjects in the HTT group added HTT movement to a regular baseball-training regimen (which included baseball technical training and some strength training without lower extremity training during the study period); participants in the CON group performed only the regular baseball training regimen for 8 weeks. Neither group had prior experience of HTT. However, members of both groups had at least 1 year of experience for squat training. In addition, the squat movement was removed from regular training one month before the HTT was initiated. Squat strength (1RM), HT strength (3RM), vertical jump, standing long jump, and 30-m sprint were assessed before and after the 8-week training program.

\subsection{Subjects}

In total, 20 recreational-level college baseball players were recruited for the current study. Subjects were moderately trained athletes with at least 2-year experience in baseball training and more than 1-year experience in weight training. They were randomly divided into the HTT group $(N=10)$ (height: $173.1 \pm$ $4.6 \mathrm{~cm}$; weight: $69.8 \pm 11.6 \mathrm{~kg}$; age: $19.9 \pm 0.8$ years) and CON group $(N=10)$ (height: $175.2 \pm 6.2 \mathrm{~cm}$; weight: $69.3 \pm 15.5 \mathrm{~kg}$; age: $20.4 \pm 2.1$ years). All subjects were injury-free during the testing and training period. The study was carried out according to the Declaration of Helsinki protocol, and written informed consent was obtained from all subjects.

\subsection{Procedures}

The participants were familiarized with the training procedures and testing movements 2-3 days before the orientation for baseline testing. They were instructed by a certified strength coach on how to perform squat and HT movements correctly. After this familiarization period, they performed a 10-minute lower-body dynamic warm-up before power exercise testing. This testing included vertical jump, long jump, and sprint. On the following day, after the 10-minute lower-body dynamic warm-up, the subjects were assessed for 1RM squat and 3RM HT. The testing procedure was repeated 3-4 days after the 8-week training program.

\subsection{Dynamic Warm-up}

The 10-minute lower-body dynamic warm-up referred to in this article consisted of one set of ten repetitions or steps of the following movements: hip, knee, and ankle mobility exercises, forward lunge, side walking with superband, butt kick, calf rise, body-weight HT, and body-weight squat.

\subsection{Vertical and Long Jumps}

To record the data of vertical jump height, the participants were asked to touch a red ink mark with the middle finger of the dominant hand and reach a maximum vertical height on the wall while their feet were flat on the ground. Subjects then were instructed 
to jump as high and as fast as they could and attempt to make contact with the middle finger at the highest possible point on the wall; the difference between the ink marks was recorded as the vertical jump height. Horizontal jump distance was measured by calculating the difference between the starting heel position and the landing heel position of the most rearward landing foot [13]. Subjects were given 3 trials to reach their maximum height and distance. Rest intervals among trials were 3 minutes. The best performance of the 3 trials was used as the comparative measure from pre-test to post-test.

\subsection{0-m Sprint}

Sprint speed over $30 \mathrm{~m}$ was assessed using an electronic timing system (Brower Timing Systems, Draper, UT). Subjects completed three trials with the fastest time recorded. Rest intervals between trials were 5 minutes.

\subsection{One Repetition Maximum Squat Strength Testing}

The 1RM squat protocol involved a progressive increase in load and decrease in repetitions per set [14]. The attempts of 1RM squats were selected with the goal of reaching the maximum for each subject in three attempts after warm-up. In between the attempts, 4 minutes of rest was permitted. During the back squat, subjects' feet were slightly wider than shoulder width apart, with toes pointed forward or slightly outward. Subjects descended until the tops of the thigh were parallel with the floor.

\subsection{Three Repetition Maximum Hip Trust Strength Testing}

After 10 minutes of rest following squat strength testing, subjects performed three progressively heavier specific warm-up sets for the barbell HT. The procedure for testing was according to Contreras et al. [13]. The barbell HT was performed by having the upper backs of subjects on a bench (Fig. 1). The feet of subjects were at a distance slightly wider than shoulder width, with toes pointed forward or slightly outward. The barbell was padded with a thick bar pad and placed over the hips of the subjects. Subjects were instructed to thrust the bar upward while maintaining a neutral position of the spine and pelvis.

All subjects had at least 1-year experience of performing the squat movement, while none had experience with the HT movement. Therefore, 3RM in HT strength testing was selected rather than 1RM because of safety concerns.

\subsection{Hip Thrust Training Protocol}

Prior to each formal training session, the participants in HTT group were instructed to perform low-load gluteus activation exercises, which included single-leg bridges, clamshells, quadruped hip extensions, and side walking with super bands. One set of ten repetitions or steps was performed for each exercise. The participants were trained by a certified strength coach and were
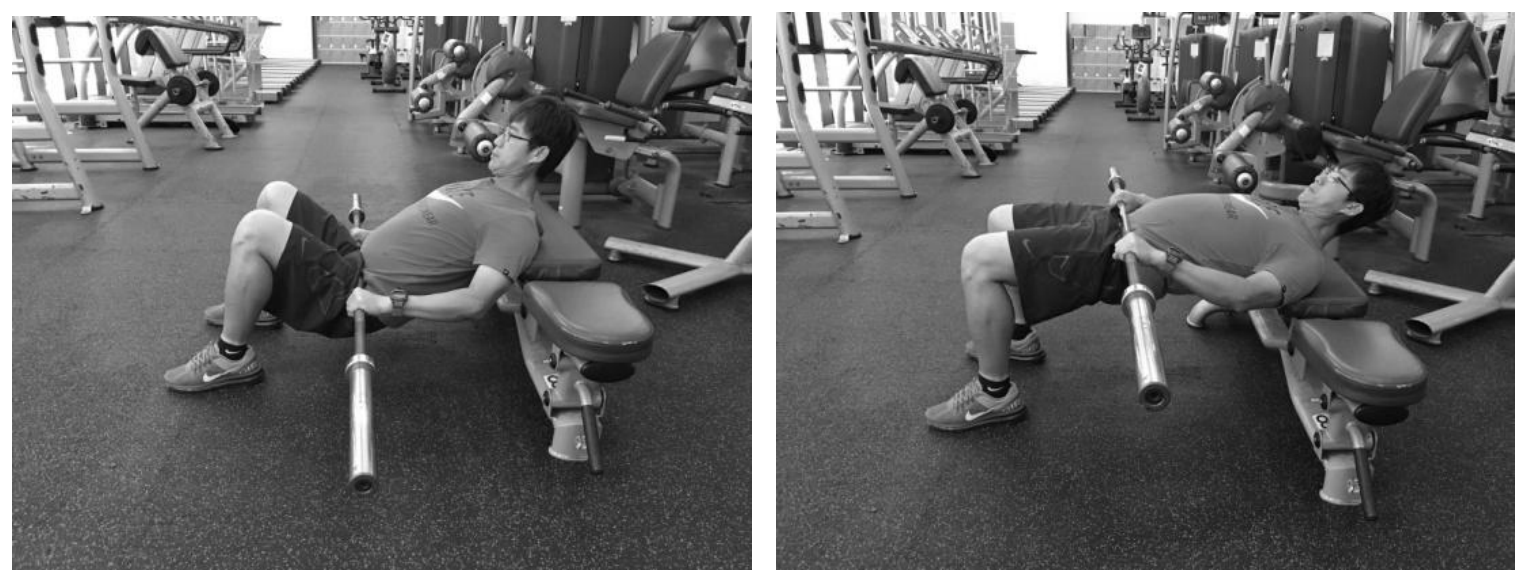

Fig. 1 Hip thrust movement from starting position (left) to ending position (right). 
taught how to maintain good control of hip-dominated movements during each training session.

The detailed description of the HTT program is presented in Table 1. The 8-week training program included three stages, which focused on enhancing endurance, hypertrophy, and strength of the hip extensors in sequence. We designed the progressive training program according to published guidelines of the American College of Sport Medicine [15]. During the training period, the subjects were instructed to maintain their usual daily activities and diet. No additional training of the lower extremities was performed during the 8 weeks. Following the 8 weeks of training, the participants rested at least 3 days after the last workout day of the program.

\subsection{Statistical Analyses}

Data are expressed as mean \pm standard deviation (SD). Baseline characteristics were assessed using the independent sample t-test. An independent sample t-test was also used to compare percentage changes in outcomes between groups from pre-training to post-training. Additionally, to determine the relationship between squat strength gains and HT strength gains in response to training, the percentage of changes in these values of the training group was analyzed using Pearson's product correlations. Statistical significance was accepted at $P<0.05$ for all tests.

\section{Results}

As shown in Table 2, there was no significant difference between groups in any of the baseline variables $(P>0.05)$. After training, significant $(P<$ 0.05 ) improvements in squat strength and 3RM HT strength were found, and the change in squat strength $(31 \% \pm 15 \%)$ positively correlated with that in $3 \mathrm{RM}$ HT strength $(36 \% \pm 16 \%)(r=0.83, P<0.05)$. However, no significant improvements were found for vertical jump, long jump, and 30 -m sprint $(P>0.05)$. Furthermore, no significant changes were observed in any of the measured variables in the CON group.

Table 1 The 8-week hip thrust training program.

\begin{tabular}{lllllllll}
\hline Week & 1 & 2 & 3 & 4 & 5 & 6 & 7 & 8 \\
\hline Load & $50 \%$ & $60 \%$ & $70 \%$ & $80 \%$ & $80 \%$ & $85 \%$ & $85 \%$ & $90 \%$ \\
(3RM) & 20 & 15 & 15 & 12 & 12 & 10 & 8 & 6 \\
Repetitions & 4 & 4 & 4 & 4 & 4 & 4 & 4 & 4 \\
Sets & 1 & 1 & 1 & 1.5 & 1.5 & 2 & 2 & 2.5 \\
Rest time (minutes) & & &
\end{tabular}

Table 2 Changes in the strength and power performance from pre- to post-training and differences among groups.

\begin{tabular}{|c|c|c|c|c|c|c|c|}
\hline & \multicolumn{3}{|c|}{$\operatorname{HTT}(N=10)$} & \multicolumn{3}{|c|}{$\mathrm{CON}(N=10)$} & \multirow{2}{*}{$\begin{array}{l}\text { Change percentage } \\
\text { between-group } \\
\text { difference }\end{array}$} \\
\hline & Pre & Post & $\begin{array}{l}\text { Change } \\
\text { percentage }\end{array}$ & Pre & Post & $\begin{array}{l}\text { Change } \\
\text { percentage }\end{array}$ & \\
\hline $\mathrm{VJ}(\mathrm{cm})$ & $56.20 \pm 7.13$ & $57.00 \pm 7.16$ & 0.59 & $59.00 \pm 8.77$ & $59.59 \pm 8.40$ & 0.74 & 0.15 \\
\hline $\mathrm{SLJ}(\mathrm{cm})$ & $260.50 \pm 17.75$ & $262.00 \pm 17.68$ & -0.25 & $262.60 \pm 17.96$ & $264.70 \pm 20.66$ & -1.50 & 1.25 \\
\hline 30-m sprint (sec) & $4.21 \pm 0.27$ & $4.19 \pm 0.21$ & -0.25 & $3.99 \pm 0.58$ & $3.91 \pm 0.46$ & 2.63 & 2.88 \\
\hline Squat (kg) & $84.00 \pm 24.59$ & $107.50 \pm 23.60$ & $30.77 *$ & $87.50 \pm 23.36$ & $89.50 \pm 22.91$ & 2.63 & $28.14^{\#}$ \\
\hline HT (kg) & $134.00 \pm 43.58$ & $178.00 \pm 44.73$ & $36.05^{*}$ & $130.00 \pm 22.36$ & $133.00 \pm 18.14$ & 3.18 & $32.87^{\#}$ \\
\hline
\end{tabular}

Values are presented as Mean \pm SD. HTT: Hip Thrust Training Group; CON: Control Group; VJ: Vertical jump; SLJ: Standing long Jump; HT: Hip Thrust.

*Significant difference between pre- and post-training.

\# Significant difference in between-group change (percentage). 


\section{Discussion}

The current study investigated the effects of 8 weeks of HTT on the strength and power performance in lower limbs of college baseball players. The principal findings of this study were that this training program can significantly enhance HT strength and squat performance, and the increments in HT strength and squat performance highly correlated $(\mathrm{r}=0.83)$. However, this training program failed to improve jump and sprint performance.

Specific strength can be enhanced by a regular resistance training program that targets a certain muscle or movement [16]. The current study employed an 8-week HTT program, including muscular endurance training using light loads and high repetitions for the first 3 weeks, for the purpose of establishing hip movement control and hip extensor endurance. The load was increased and the repetitions decreased gradually for the next 5 weeks, which was reflected during the transition of the training goal from the muscle hypertrophy phase to the final goal of strength gain. As expected, the HT 3RM strength increased after 8 weeks of training. The increment was $36.05 \%$ on an average. This finding is similar to that of Contreras et al. [13], who found a significant increase (29.95\%) in HT 3RM strength after 6 weeks of HTT.

The outcome measure of squat performance also improved after our training program. It is noteworthy that the participants in our study had experienced at least 1 year of squat training, but the squat movement was removed from regular training one month before the HTT was initiated. In this study, following the HTT, back squat strength greatly increased showing a $30.77 \%$ improvement on an average. The result is similar to that of Contreras et al. [13], who demonstrated a $6.63 \%$ improvement on an average in front squat strength after 6 weeks of HTT. The greater improvement in squat strength in our study than that found by Contreras et al. [13] may be attributed to the difference in assessment based on the squat types (back squat vs. front squat). Previous studies indicated that the ratio of hip-to-knee extensor moments increases with increasing load during back squats [17]. This implies that the hips occupy a more important role as the load of squats becomes heavier. Moreover, individuals often exhibited a greater trunk lean for the back squat relative to the front squat [18], suggesting that back squats load the hips more than front squats. Therefore, the current study addressed the importance of hip training while developing back squat strength, and it is speculated that HT is more favorable for developing back squat strength than front squat. In addition, the training frequency and the total number of training sessions should also be considered. Unlike our study, which employed 36 training sessions, there were only 12 training sessions ( 6 weeks of biweekly) in the Contreras's study. Similar but lesser increments were found by Lake et al. [12], who reported a $12.03 \%$ improvement in back squat strength after 6 weeks of biweekly kettlebell swing exercise, which also addresses the hip extensor muscles.

The improvement of hip extensor strength was initially theorized to result in better power performance of the lower extremities. Although hip extensors are highly activated during sprint and functionally important for powerful movements [19], there were no changes in jumping and sprint performance after HTT in our study. These results disagree with previous studies that emphasized on hip extension training movement using either HT or kettlebell swing exercise $[12,13]$. A possible explanation for the divergent results could be the differences in both the mode used and the subject characteristics. Regarding the training mode because of safety concerns, our participants were instructed to lift the barbell at moderate speed (1 second up, hold 1 second, and 1 second down). However, it has been suggested that movement speed occupies the central role when the training goal is to enhance explosive performance $[20,21]$. This is termed as a velocity-specific adaptation. The associated neural adaptations may include the selective 
activation of motor units and/or muscles, especially with high-velocity alternating contractions. The HTT in our current study lacked explosive movement, which may not fit the training specificity for developing power performance. In addition, the training load in our study started at 20RM in the first week and gradually increased load to 6RM at the last week. Traditionally, 1-6RM, 8-12RM, and 15-25RM loads are best trained to maximize strength, hypertrophy, and muscular endurance, respectively [22]. It appears that there was a limited power-specific training phase in our training mode. Regardless of movement speed, it should be noted that HTT lacks in complex coordination. It is likely that explosive performance is determined by a more complex coordination of triple extension of the hips, knees, and ankles. Therefore, our HTT mode, which only addressed hip extension, lacked specificity and thus may not have improved explosive performance. Although the HT did not enhance power performance effectively in our study, significant increase in jump and sprint performance were observed by Contreras et al. [13]. In the Contreras study they recruited adolescent subjects. Scientific evidence indicates that adolescents tend towards greater absolute explosive power capacity gains due to growth and maturation [23]. Experienced adult baseball players, such as our subjects, may have less strength-training improvement in power performance than adolescents

Limitation regarding the interpretation of this study should be mentioned. In general, a strength and conditioning program is often designed periodically for optimize specific muscle performance, including hypertrophy, strength and power training stages in sequence. Because the HT training is a novel exercise, the training program in the presented study was mainly followed general training guidelines, that is, using a moderate to high loads with slow to moderate velocities [8, 13], but lack of dynamic movement. Since movement- and velocity specificity may occupy an important consideration for effectiveness in interventions designed to improve power performance outcomes, thus, further research is required to add power training element to the HT training program. Despite of this limitation, the findings provide useful information on strength improvement to HTT.

\section{Conclusion}

In conclusion, although the HTT program used in the present study was neural to the power performance, the back squat strength as well as HT strength was dramatically increased, which may somewhat assist in transferring strength to power performance later. It is further suggested that future HT training study should include power training element in the training program to clarify the effect on power performance.

\section{Acknowledgements}

No financial assistance or grant support was obtained for this study. The authors appreciate the contribution of all participants.

\section{References}

[1] Bartlett, J. L., Sumner, B., Ellis, R. G., and Kram, R. 2014. "Activity and Functions of the Human Gluteal Muscles in Walking, Running, Sprinting, and Climbing." American Journal of Physical Anthropology 153 (1): 124-31.

[2] Lieberman, D. E., Raichlen, D. A., Pontzer, H., Bramble, D. M., and Cutright-Smith, E. 2006. "The Human Gluteus Maximus and Its Role in Running." Journal of Experimental Biology 209 (Pt 11): 2143-55.

[3] Steele, J., Bruce-Low, S., and Smith, D. A. 2015. "Review of the Specificity of Exercises Designed for Conditioning the Lumbar Extensors." British Journal of Sports Medicine 49 (5): 291-7.

[4] Kankaanpää, M., Taimela, S., Laaksonen, D., Hänninen, O., and Airaksinen, O. 1998. "Back and Hip Extensor Fatigability in Chronic Low Back Pain Patients and Controls." Archives of Physical Medicine and Rehabilitation 79 (4): 412-7.

[5] Leinonen, V., Kankaanpää, M., Airaksinen, O., and Hänninen, O. 2000. "Back and Hip Extensor Activities during Trunk Flexion/extension: Effect of Low Back Pain and Rehabilitation." Archives of Physical Medicine and Rehabilitation 81 (1): 32-7.

[6] Powers, C. M. 2010. "The Influence of Abnormal Hip Mechanics on Knee Injury: A Biomechanical Perspective.” Journal of Orthopaedic and Sports Physical Therapy 40 (2): 42-51. 
[7] Nadler, S. F., Malanga, G. A., DePrince, M., Stitik, T. P., and Feinberg, J. H. 2000. "The Relationship between Lower Extremity Injury, Low Back Pain, and Hip Muscle Strength in Male and Female Collegiate Athletes." Clinical Journal of Sport Medicine 10 (2): 89-97.

[8] Contreras, B., Cronin, J., and Schoenfeld, B. 2011. "Barbell Hip Thrust." Strength and Conditioning Journal 33 (5): 58-61.

[9] Beardsley, C., and Contreras, B. 2014. "The Increasing Role of the Hip Extensor Musculature with Heavier Compound Lower-body Movements and More Explosive Sport Actions." Strength and Conditioning Journal 36 (2): 49-55.

[10] Eckert, R. M., and Snarr, R. L. 2014. "Barbell Hip Thrust." Journal of Sport and Human Performance 2 (2): 1-9.

[11] Contreras, B. M., Cronin, J. B., Schoenfeld, B. J., Nates, R. J., and Sonmez, G. T. 2013. "Are All Hip Extension Exercises Created Equal?" Strength and Conditioning Journal 35 (2): 17-22.

[12] Lake, J. P., and Lauder, M. A. 2012. "Kettlebell Swing Training Improves Maximal and Explosive Strength." Journal of Strength and Conditioning Research 26 (8): 2228-33.

[13] Contreras, B., Vigotsky, A. D., Schoenfeld, B. J., Beardsley, C., McMaster, D. T., Reyneke, J., and Cronin, J. 2016. "Effects of a Six-week Hip Thrust versus Front Squat Resistance Training Program on Performance in Adolescent Males: A Randomized-controlled Trial." Journal of Strength and Conditioning Research [Epub ahead of print].

[14] Joy, J. M., Oliver, J. M., McCleary, S. A., Lowery, R. P., and Wilson, J. M. 2013. "Power Output and Electromyography Activity of the Back Squat Exercise with Cluster Sets." Journal of Sports Science 1 (1): 37-45.

[15] Garber, C. E., Blissmer, B., Deschenes, M. R., Franklin, B. A., Lamonte, M. J., Lee, I. M., Nieman, D. C., and Swain, D. P. 2011. "American College of Sports Medicine Position Stand. Quantity and Quality of Exercise for
Developing and Maintaining Cardiorespiratory, Musculoskeletal, and Neuromotor Fitness in Apparently Healthy Adults: Guidance for Prescribing Exercise. American College of Sports Medicine." Medicine and Science in Sports and Exercise 43 (7): 1334-59.

[16] Folland, J. P., and Williams, A. G. 2007. "The Adaptations to Strength Training: Morphological and Neurological Contributions to Increased Strength." Sports Medicine 37 (2): 145-68.

[17] Beardsley, C., and Contreras, B. 2014. "The Increasing Role of the Hip Extensor Musculature with Heavier Compound Lower-body Movements and More Explosive Sport Actions." Strength and Conditioning Journal 36 (2): 49-55.

[18] Yavuz, H. U., Erdağ, D., Amca, A. M., and Aritan, S. 2015. "Kinematic and EMG Activities during Front and Back Squat Variations in Maximum Loads." Journal of Sports Sciences 33 (10): 1058-66.

[19] Bartlett, J. L., Sumner, B., Ellis, R. G., and Kram, R. 2014. "Activity and Functions of the Human Gluteal Muscles in Walking, Running, Sprinting, and Climbing." American Journal of Physical Anthropology 153 (1): 124-31.

[20] Behm, D. G., and Sale, D. G. 1993. "Velocity Specificity of Resistance Training." Sports Medicine 15 (6): 374-88.

[21] Aagaard, P., Simonsen, E. B., Andersen, J. L., Magnusson, P., and Dyhre-Poulsen, P. 2002. "Increased Rate of Force Development and Neural Drive of Human Skeletal Muscle Following Resistance Training." Journal of Applied Physiology 93 (4): 1318-26.

[22] Spiering, B. A., Kraemer, W. J., Anderson, J. M., Armstrong, L. E., Nindl, B. C., Volek, J. S., and Maresh, C. M. 2008. "Resistance Exercise Biology: Manipulation of Resistance Exercise Programme Variables Determines the Responses of Cellular and Molecular Signalling Pathways." Sports Medicine 38 (7): 527-40.

[23] Manna, I. 2014. "Growth Development and Maturity in Children and Adolescent: Relation to Sports and Physical Activity." American Journal of Sports Science and Medicine 2 (5A): 48-50. 